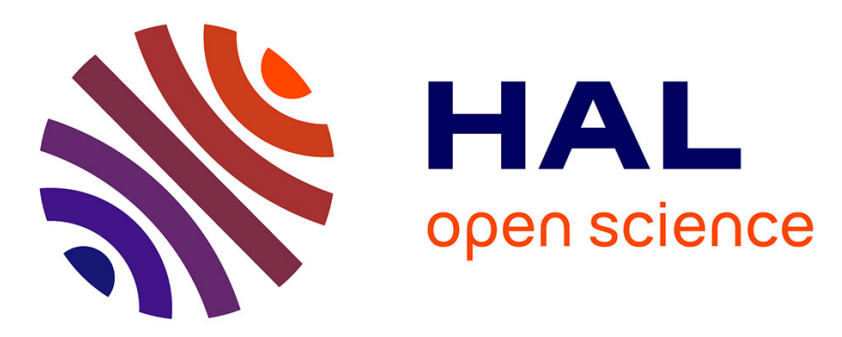

\title{
Review de: Bernard de Clairvaux, Sermons variés, texte latin des S. Bernardi Opera par J. Leclercq, H. Rochais et Ch. H. Talbot, introduction et notes par F. Callerot (Sources chrétiennes, 526)
}

Marie Pauliat

\section{To cite this version:}

Marie Pauliat. Review de: Bernard de Clairvaux, Sermons variés, texte latin des S. Bernardi Opera par J. Leclercq, H. Rochais et Ch. H. Talbot, introduction et notes par F. Callerot (Sources chrétiennes, 526). Augustinianum: periodicum quadrimestre Collegii internationalis Augustiniani, 2013, 53, pp.564-567. 10.5840/agstm201353235 . halshs-03168434

\section{HAL Id: halshs-03168434 \\ https://shs.hal.science/halshs-03168434}

Submitted on 16 Mar 2021

HAL is a multi-disciplinary open access archive for the deposit and dissemination of scientific research documents, whether they are published or not. The documents may come from teaching and research institutions in France or abroad, or from public or private research centers.
L'archive ouverte pluridisciplinaire HAL, est destinée au dépôt et à la diffusion de documents scientifiques de niveau recherche, publiés ou non, émanant des établissements d'enseignement et de recherche français ou étrangers, des laboratoires publics ou privés. 
Le volume 526 de la collection «Sources chrétiennes » complète la publication des Euvres complètes de Bernard de Clairvaux par dix Sermons variés, qui forment le numéro XXVIIIB de la série bernardine.

Le texte latin suit les mêmes principes d'établissement et de présentation que les autres volumes des œuvres complètes de Bernard. L'édition critique des Sancti Bernardi Opera procurée par Jean Leclerc, assisté de Henri Rochais et Charles H. Talbot, a bénéficié de corrections depuis sa publication; l'édition des Sources chrétiennes profite de ces amendements. Quelques corrections nouvelles apportées au texte latin sont signalées p. 25. De fait, l'apparat critique n'est pas reproduit et seules paraissent dans l'annotation les variantes davantage liées au sens du texte. L'annotation est l'œuvre de Françoise Callerot, complétée ponctuellement par le travail de Pierre-Yves Emery, Gaetano Raciti et Bernard-Joseph Samain. Les sources des citations sont, dans la mesure du possible, précisées en notes : Vulgate, Pères de l'Eglise, Liturgie ou Règle de saint Benoît. Un astérisque indique que la note explicite une référence scripturaire ; Jean Figuet et Laurence Mellerin essentiellement en sont à l'origine.

Françoise Callerot a quant à elle été la maîtresse d'œuvre de ce volume ; elle a révisé la traduction française de la quasi-totalité de ces sermons, due au frère Pierre-Yves Emery (Taizé). Elle a également rédigé l'introduction générale, les introductions propres à chaque sermon et inséré les sous-titres de la traduction. Seul fait exception le sermon BenV, traduit par son inventeur, Gaetano Raciti ; son introduction et son annotation sont redevables à un article du même auteur (CollCist 60, 1998, p. 57-107) ; le schéma qui est y est donné p. 87 été repris pour les sous-titres. Par ailleurs, trois de ces sermons variés (VicS1, VicS2 et MalV) avaient déjà été publiés dans les Sources chrétiennes ; leurs traductions et leurs annotations ont été révisées.

Les sigles et abréviations que comporte ce volume sont récapitulés avant l'introduction générale qui explique la constitution de la collection de ces Sermons variés. Certains d'entre eux ressemblent aux Sermons pour l'année liturgique, recueil dont saint Bernard, qui était avant tout un prédicateur, a lui-même retravaillé la forme littéraire ; d'autres s'apparentent aux Sermons divers que le prédicateur n'a pas revus ni intégrés à la première collection. Dans ce cas, les Sermons variés se caractérisent comme les Sermons divers par la variété des sujets, par l'absence de révision par leur auteur et par leur authenticité ; si la forme littéraire est moins achevée que dans les sermons que Bernard a lui-même publiés, sa doctrine y est cependant présente et ils font résonner la prédication quotidienne de l'abbé de Clairvaux.

Ces Sermons variés ont été transmis par des traditions manuscrites distinctes de celles des autres sermons. Six d'entre eux avaient été édités par Mabillon au XVII ${ }^{\mathrm{e}}$ siècle et répartis à tord parmi les Sermons pour l'année, et quatre étaient inédits : ceux sur l'Avent, l'Epiphanie, et les sept dons de l'Esprit ont été ajoutés par Dom Leclerc dans son édition critique ; Gaetano Raciti a, en 1998, découvert le quatrième, pour la fête de saint Benoît ; il figure naturellement dans ce volume.

Ces dix sermons ont, dans l'édition critique, été organisés en fonction du cycle liturgique et non dans l'ordre chronologique de leur prédication. Leurs titres, présents comme points de repères dans les manuscrits, ne sont pas de Bernard. Chaque sermon est précédé d'une introduction propre qui présente sa structure souvent complexe, explicite d'éventuelles difficultés thématiques (la vie de saint Victor, récapitulée à partir des œuvres de Bernard p. 128) ou formelles (par exemple, le second titre, De triplici inferno, du sermon In Adventu), met en relief des traits stylistiques et discute de son authenticité (celle du second sermon, tissé de thèmes bernardins, ne fait aucun doute, mais il est tout aussi indubitable que la rédaction décousue n'est pas de Bernard). 
Les deux premiers sermons se rapportent aux célébrations liturgiques du temporal : l'un porte sur 1'Avent - In Adventu (De triplici inferno) - et l'autre sur l'Epiphanie - In Epiphania. L'ancrage liturgique du premier sermon dans la liturgie de l'Avent, tournée vers l'avènement eschatologique du Christ qu'annoncent les prophéties, en explique le second titre ; la vie monastique, par l'enfer de la «pauvreté volontaire » qui purifie, est un moyen efficace de participer à la Rédemption qu'offre le Christ ; elle est aussi la personnification terrestre de la Cité Sainte, son modèle céleste. Même si, dans l'exil de la terre, l'Epoux semble tarder, le salut viendra bientôt. Dans le second sermon, In Epiphania, Bernard, après avoir exposé les trois manifestations du Christ (la venue des Mages, le Baptême par Jean, les noces de Cana), développe le thème du retour de l'âme vers sa Patrie, à l'instar des Mages qui regagnèrent la leur. La grâce, absolument nécessaire pour que la liberté atteigne son but qui est Dieu, ne supprime pas le combat spirituel. Heureusement, le Christ Médiateur s'offre gratuitement pour la restauration de la paix avec Dieu, avec nous-mêmes et avec le prochain. Alors, dans le moine éclairé tout entier par la sagesse et qui se livre à l'obéissance, la paix intérieure s'installe.

Les quatre sermons suivants, prêchés pour la conversion de saint Paul le 25 janvier (In conversione sancti Pauli), pour la fête locale de saint Victor le 26 février (In natali sancti Victoris sermo primus et sermo secundus) et pour la fête de saint Benoît le 21 mars (In natale sancti Benedicti), relèvent du sanctoral. Le premier sermon, plus proche du résumé, insiste sur la nécessité absolue de la conversion; sans cette intervention du Christ et cette conformation à lui, spécialement à l'humilité et à la douceur de son enfance, il est impossible de prendre place dans le Royaume. Les deux sermons pour la fête de saint Victor, composés à la demande des bénédictins de Montiéramey, se complètent : le premier est consacré à la vie du saint sur terre ; le second décrit sa gloire et sa compassion dans le ciel. Dans le banquet qu'est la vie du saint, il faut se contenter de recueillir ses exemples sans chercher à s'approprier le merveilleux qui entoure sa vie. Dans la gloire du ciel, Victor est à présent avocat et médiateur et, animé par la charité, il intercède pour les hommes avec tous les saints. Mt 19, 27-29, la péricope liturgique de la fête de saint Benoît, est intégralement commentée dans le quatrième sermon. Il relève de l'œuvre monastique de Bernard : le don total du moine se fonde sur le mystère du Verbe fait chair; la liberté spirituelle est un fruit de l'adoption divine. En revanche, le prédicateur ne dit mot ni du cloître, ni du lien privilégié du moine avec le Christ, sur le modèle des apôtres («vie apostolique »). Ce sermon est authentique jusque dans sa rédaction.

Enfin, les quatre derniers sermons, qui n'appartiennent pas au cycle liturgique, ont un sujet précis : la volonté de Dieu (De voluntate divina); les miséricordes du Seigneur (De misericordiis); les sept dons du Saint Esprit (De septem doni Spiritus Sancti); la Pâque de saint Malachie (De sancto Malachia). Si nous demandons, dans la troisième demande du Pater que commente Bernard dans le sermon De voluntate divina, que notre volonté soit unie à celle de Dieu, c'est que ce n'est pas encore le cas ; seule la grâce divine peut restaurer les quatre vertus cardinales qui rendent victorieux des vices. La grâce est nécessaire au salut et, après avoir prévenu l'homme, elle l'accompagne tout au long de son cheminement terrestre jusqu'à se transformer en gloire dans le ciel pour celui qui l'a accueillie. Tel est le principe sur lequel repose le sermon De misecordiis, qui recommande aux moines susceptibles de se replier sur eux-mêmes, de rendre grâce pour les miséricordes reçues du Seigneur. Sur le ton du récit, Bernard reconstitue l'usage que le Christ a fait des sept dons de l'Esprit pour le salut de l'homme : la «théologie monastique » s'efforce, dans ce troisième sermon, d'identifier la convenance des actions du Christ, sujet traité par la comparaison avec les cinq sens corporels dans le cas des dons de sagesse et d'intelligence. Enfin, la relation chaleureuse entre l'abbé de 
Clairvaux et le moine Irlandais Malachie transparaît à travers les thèmes du dernier sermon : la justification, don de Dieu; la coopération à l'œuvre de Dieu qu'a réalisée Malachie ; la gratuité de son amour ; le dynamisme de son « œil simple »; sa présence à Dieu et aux autres.

Un index scripturaire complète le volume; il distingue les citations des allusions et dissocie les références des versets conformes à la Vulgate de celles des versets dont le texte est spécifiquement bernardin.

Ce volume des « Sources Chrétiennes » offre donc, dans une traduction claire et précise, un accès commode à des textes auparavant dispersés dans différentes éditions, textes qui, s'ils ne comptent parmi les œuvres majeures de Bernard, complètent toutefois fort heureusement notre connaissance de l'abbé de Clairvaux et de sa prédication ordinaire. 